\title{
Deducing transport properties of mobile vacancies from perovskite solar cell characteristics
}

James M. Cave, ${ }^{1}$ Nicola E. Courtier, ${ }^{2}$ Isabelle A. Blakborn, ${ }^{3}$ Timothy W. Jones, ${ }^{3}$ Dibyajyoti Ghosh, ${ }^{4}$ Kenrick F Anderson, ${ }^{3}$ Liangyou Lin, ${ }^{3}$ Andrew A. Dijkhoff, ${ }^{3}$ Gregory J. Wilson, ${ }^{3}$ Krishna Feron, ${ }^{3}$ M. Saiful Islam, ${ }^{4}$ Jamie M. Foster, ${ }^{5}$ Giles Richardson, ${ }^{2}$ and Alison B. Walker ${ }^{6, \text { a) }}$

${ }^{1)}$ Department of Physics, University of Bath, BA2 7AY, UK

2) Mathematical Sciences, University of Southampton, SO17 1BJ, UK

${ }^{3)}$ CSIRO Energy, Newcastle Energy Centre, 10 Murray Dwyer Circuit, Mayfield West, NSW 2304, Australia ${ }^{\mathrm{b})}$

4) Department of Chemistry, University of Bath, BA2 $7 A Y, U K^{\mathrm{c})}$

${ }^{5)}$ Department of Mathematics, University of Portsmouth, PO1 $3 H F$, UK

${ }^{6}$ Department of Physics, University of Bath, BA2 7AY, UK

(Dated: 13 October 2020)

The absorber layers in perovskite solar cells possess a high concentration of mobile ion vacancies. These vacancies undertake thermally activated hops between neighbouring lattice sites. The mobile vacancy concentration $N_{0}$ is much higher and the activation energy $E_{A}$ for ion hops is much lower than is seen in most other semiconductors due to the inherent softness of perovskite materials. The timescale at which the internal electric field changes due to ion motion is determined by the vacancy diffusion coefficient $D_{v}$ and is similar to the timescale on which the external bias changes by a significant fraction of the open circuit voltage at typical scan rates. Therefore hysteresis is often observed in which the shape of the current-voltage, J-V, characteristic depends on the direction of the voltage sweep. There is also evidence that this defect motion plays a role in degradation. By employing a charge transport model of coupled ion-electron conduction in a perovskite solar cell, we show that $E_{A}$ for the ion species responsible for hysteresis can be obtained directly from measurements of the temperature variation of the scan-rate dependence of short-circuit current and of the hysteresis factor $H$. This argument is validated by comparing $E_{A}$ deduced from measured $\mathrm{J}-\mathrm{V}$ curves for four solar cell structures with density functional theory calculations. In two of these structures the perovskite is $\mathrm{MAPbI}_{3}$ (MAPI) where MA is methylammonium, $\mathrm{CH}_{3} \mathrm{NH}_{3}$, the hole transport layer (HTL) is spiro (spiro-OMeTAD, 2, ', , 7, 7'tetrakis[N,N-di(4-methoxyphenyl) amino]-9,9'-spirobifluorene) and the electron transport layer (ETL) is $\mathrm{TiO}_{2}$ or $\mathrm{SnO}_{2}$. For the third and fourth structures, the perovskite layer is $\mathrm{FAPbI}_{3}$ (FAPI) where FA is formamidinium, $\mathrm{HC}\left(\mathrm{NH}_{2}\right)_{2}$, or $\mathrm{MAPbBr}_{3}(\mathrm{MAPBr})$ and in both cases the HTL is spiro and the ETL is $\mathrm{SnO}_{2}$. For all four structures, the hole and electron extracting electrodes are Au and FTO (fluorine doped tin oxide) respectively. We also use our model to predict how the scan rate dependence of the power conversion efficiency varies with $E_{A}, N_{0}$ and parameters determining free charge recombination.

Keywords: solar cell, perovskite, electrical characteristics, mixed ionic-electronic conduction, measurements, simulation, hysteresis, density functional theory, drift diffusion

\section{INTRODUCTION}

Perovskite solar cells (PSCs) have generated intense research interest since the power conversion efficiencies (PCEs) of laboratory cells now match those of the best established inorganic polycrystalline technologies due to the rapid rise in power conversion efficiency and they are easy to make in the lab through low-temperature solution processing ${ }^{1,2}$ due to their soft nature ${ }^{3,4}$. Other advantages include high absorptivity and excellent carrier diffusion lengths ${ }^{5}$.

Recent studies, for example electro-absorption measurements ${ }^{6}$, multidimensional luminescence imaging ${ }^{7}$ and transfer curves and output curves of perovskite field effect transistors ${ }^{8}$, have demonstrated ion motion occurs in

a) Electronic mail: email a.b.walker@bath.ac.uk

b) Also at Faculty of Applied Sciences, Delft University of Technology, 2628 CJ Delft, The Netherlands

c) Also at Department of Physics, University of Bath, BA2 7AY, UK perovskite devices. Ion motion can be beneficial, such as in memristors ${ }^{9}$ and it can enhance PSC performance ${ }^{10}$. While mobile ions are not always associated with less stable devices, they have been shown to impose constraints on cell performance and stability assessment ${ }^{11}$ and can result in a lower efficiency with the same concentration of defects compared to the same device without ions ${ }^{12}$. Furthermore, vacancy migration accelerates non radiative charge recombination by inducing lattice distortions ${ }^{13}$ and perovskite layers and their interfaces with transport layers are degraded by the strain in the perovskite lattice ${ }^{14}$.

In standard measurements of hysteresis, a reverse scan that sweeps the applied bias from just above the open-circuit voltage $\left(V_{o c}\right)$ to zero volts, is followed by a forward scan taking the applied bias from zero volts (at which the short-circuit current density $\left(J_{s c}\right)$ is measured) to the voltage at the start of the reverse scan. For a given applied bias, the currents measured on the reverse scan are greater (normal hysteresis) or less (inverted hysteresis ${ }^{15}$ ) than those measured on the forward scan. To quantify these effects, the hysteresis factor $H=\left(A_{\text {rev }}-A_{\text {for }}\right) / A_{\text {rev }}$, where $A_{\text {rev }}$ and $A_{\text {for }}$ are the areas un- 
(a)

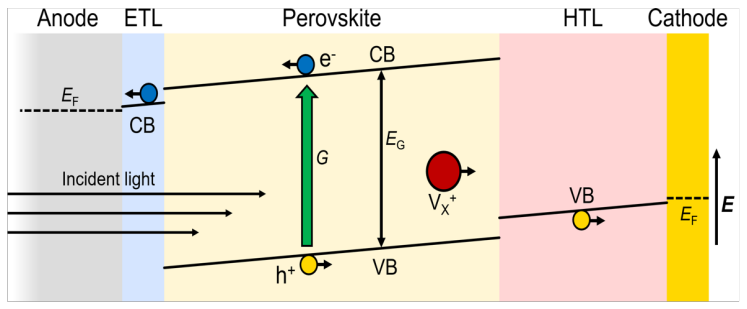

(b)

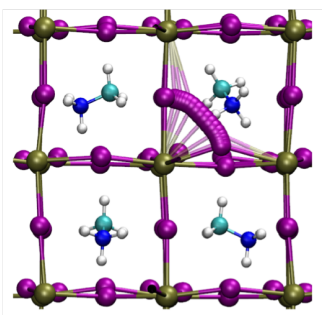

FIG. 1. a Planar PSC structure. Its materials composition for structures a-d is defined in the text. The simulated ETL, perovskite layer and HTL thicknesses for the four device architectures, whose measured and modelled characteristics are reported in this paper, are $50 \mathrm{~nm}, 400 \mathrm{~nm}$ and $200 \mathrm{~nm}$ respectively. The green vertical arrow shows generation of electrons ( ${ }^{-}$, blue discs) and holes $\left(\mathrm{h}^{+}\right.$, yellow discs) through light absorption in the perovskite layer. Carriers are then transported as shown by horizontal arrows to the carrier-selective, electron and hole, transport layers (ETL and HTL, respectively) and from there into the electrodes. Conduction and valence bands (CB and VB), the band gap $\mathrm{E}_{G}$, Fermi levels $\left(E_{F}\right)$ and a halide vacancy $\left(\mathrm{V}_{\mathrm{X}}^{+}\right.$, red disc) are shown. b Migration path of $\mathrm{I}^{-}$vacancy in MAPI from our DFT simulations. The colour scheme is: purple, $\mathrm{I}^{-}$; dark blue, $\mathrm{N}$; cyan, $\mathrm{C}$; white, $\mathrm{H}$; and brown, $\mathrm{Pb}$.

der the current-voltage (J-V) curve for reverse and forward scan respectively, is used. The hysteresis observed in our cells is the normal kind in which $A_{\text {rev }}>A_{\text {for }}$ (so that $H>0$ ), however inverse or mixed hysteresis is also possible and in such cases the definitions suggested by Nemnes et al ${ }^{16}$ may be more appropriate. The hysteresis factor $H$ is important because it can be used to infer the timescales associated to defect motion. In particular, it takes its largest values at scan rates where changes in the internal field due to change in bias are comparable to internal field changes from ion motion over the same time period. Cell characteristics, such as $H$, that encapsulate kinetic effects should be used with care because of their sensitivity to scan speed ${ }^{17,18}$, scan range, scan direction, prebiasing, and temperature ${ }^{19}$.

It is also important to note that $A_{\text {rev }}$ and $A_{\text {for }}$ do not measure the power generated during those scans since a $\mathrm{J}-\mathrm{V}$ curve describes a locus of operational states

A PSC structure and its operation are shown in Figure 1a. Measured $\mathrm{J}-\mathrm{V}$ curves at varying scan rates and temperatures are reported here for four PSC structures labelled a,b,c,d. In structures $a$ and $b$, the light harvesting layer is $\mathrm{MAPbI}_{3}$ (MAPI), in structure $\mathrm{c}$ it is $\mathrm{FAPbI}_{3}$ (FAPI) and in structure d, it is $\mathrm{MAPbBr}_{3}(\mathrm{MAPBr})$. For these perovskites, the organic cation is methylammonium $\left(\mathrm{MA}, \mathrm{CH}_{3} \mathrm{NH}_{3}\right.$ ) or formamidinium $\left(\mathrm{FA}, \mathrm{HC}\left(\mathrm{NH}_{2}\right)_{2}\right)$. In all four PSC architectures the hole transport layer (HTL) is spiro (spiro-OMeTAD, 2,2',7,7' -tetrakis[N,N-di(4methoxyphenyl)amino]-9,9'-spirobifluorene). The electron transport layer (ETL) is $\mathrm{TiO}_{2}$ in structure a, and $\mathrm{SnO}_{2}$ for the other structures. In all four structures the hole and electron extracting electrodes are Au and FTO (fluorine doped tin oxide) respectively.

For perovskites, Schottky defects dominate. These defects are unoccupied anion and cation sites in a stoichiometric ratio and are randomly distributed in a crystal in equilibrium. At full Schottky disorder, the unoccupied anion sites are positively charged iodide vacancies, $\mathrm{V}_{I}^{+}$, and unoccupied cation sites are negatively charged MA vacancies, $\mathrm{V}_{M A}^{-}$, and $\mathrm{Pb}$ vacancies, $\mathrm{V}_{P b}^{-20}$ Density functional theory (DFT) calculations for MAPI predict a low Schottky formation energy per defect $E_{V}$ of $0.14 \mathrm{eV},{ }^{20}$, consistent with $0.1-0.2 \mathrm{eV}$ reported by Tong et $\mathrm{al}^{21}$. At room temperature, the $\mathrm{V}_{I}^{+}$concentration $N_{0}=$ $0.4 \%=1.6 \times 10^{19} \mathrm{~cm}^{-3}$ for a cubic unit cell with lattice constant $a=0.628 \mathrm{~nm},{ }^{22,23}$. Eames et $\mathrm{al}^{22}$ estimated $E_{A}$ for $\mathrm{V}_{I}^{+}$as $0.58 \mathrm{eV}$ for cubic MAPI (similar to the more recent value of $0.55 \mathrm{eV}^{24}$ ). They find a much higher $E_{A}$ of $0.84 \mathrm{eV}$ for $\mathrm{V}_{M A}^{-}$ migration, given that its path is through the unit cell face or through a bottleneck comprising four $\mathrm{I}^{-}$ions, and $E_{A}$ of 2.31 $\mathrm{eV}$ for $\mathrm{V}_{P b}^{-}$, suggesting an immobile $\mathrm{Pb}$ sublattice.

We have predicted the $\mathrm{V}_{I}^{+}$trajectory between adjacent lattice sites in tetragonal MAPI, shown in Figure 1b and note it is similar in shape to the path reported by Eames et $\mathrm{al}^{22}$. The diffusion coefficient of ion vacancies $D_{v}$ has an Arrhenius dependence on temperature $T, D_{v}=D_{v \infty} \exp \left(-E_{A} / k_{B} T\right)=$ $2 \times 10^{-13} \mathrm{~cm}^{2} / \mathrm{s}$ at room temperature for $E_{A}=0.58 \mathrm{eV}$. Here the 
high temperature ion diffusion coefficient $D_{v \infty}=a^{2} f_{a} / 12=$ $3 \times 10^{-4} \mathrm{~cm}^{2} \mathrm{~s}^{-1}$ where $f_{a}=1 \mathrm{THz}$ is the hop attempt frequency.

To see hysteresis in J-V scans, the timescale at which ion motion influences fields within the perovskite layer should be comparable to the time taken by the J-V scan as measured by the scan rate. Ion motion causes local accumulation of space charge in narrow layers termed Debye layers. For experimental measurements of $\mathrm{J}-\mathrm{V}$ curves, $\mathrm{V}_{I}^{+}$is the only species whose migration occurs on the relevant time scales. The Debye layer created by accumulation of $\mathrm{V}_{I}^{+}$next to the HTL has width $L_{D} \approx 2 \mathrm{~nm}$ using parameter values in Table II of the Supplementary Material, SM. A second wider Debye layer next to the ETL arises from $\mathrm{V}_{I}^{+}$depletion ${ }^{19,22,23,25-27}$. The Debye layers alter the electrostatic potential profile which is not significantly affected by the free charge carriers whose concentration, even at the maximum value at open circuit, is four orders of magnitude lower than $N_{0}$.

The following argument ${ }^{28}$ gives a rough estimate for these timescales. The ion mobility deduced from $D_{v}$ for $\mathrm{V}_{I}^{+}$using the Einstein relation gives a drift velocity of $2 \times 10^{-7} \mathrm{~cm} / \mathrm{s}$ at short circuit, assuming a field of $V_{o c} / b$ where $b=400 \mathrm{~nm}$ is the perovskite layer width. Therefore it takes about $1 \mathrm{~s}$ for ions to move a distance equal to $L_{D}$ and hence for charge to be transferred between the two perovskite Debye layers due to all mobile ions moving an average distance of one Debye length. This timescale is consistent with maximum hysteresis occurring at scan rates of around a few hundred $\mathrm{mV} / \mathrm{s}$ and with the low frequency semi-circles observed in Nyquist plots of impedance measurements. These semicircles can be interpreted as arising from modifications in the rate of charge recombination caused by ion motion ${ }^{28}$.

Timescales of around 6 hours have been associated with the motion of the methylammonium vacancy $\mathrm{V}_{M A}^{-}{ }^{14}$, and since these are much greater than the timescales observed in our measurements we do not explicitly model the motion of such defects, instead modelling them as a uniformly distributed static background counter charge.

The effects of ion migration on charge carrier injection or extraction at interfaces of this layer with the charge transport layers, included in our model, can also be important ${ }^{29}$. The band alignment of the semiconductor with the electrode work functions is changed by the Debye layers, modifying current injection and electron-hole recombination ${ }^{28,30,31}$.

We have developed a predictive transport model that considers ion motion. This model shows charge build up at the perovskite/charge transport layer interfaces due to ion motion that causes electrostatic potential changes in the perovskite layer and the HTL and ETL, interface recombination, band offsets at the perovskite/charge transport layer interfaces and free charge carrier transport in the HTL and ETL as well as the perovskite layer. A description of the code and a free copy can be downloaded from ${ }^{32}$.

In this article we use our model, where $E_{A}$ is fixed at 0.58 $\mathrm{eV}$, to predict and explain $\mathrm{J}-\mathrm{V}$ curves at three scan rates and $H$ and $J_{s c}$ vs scan rate and temperature. These predictions are validated by comparing with experimental measurements for structure a. Then we show $E_{A}$ can be extracted from measure-
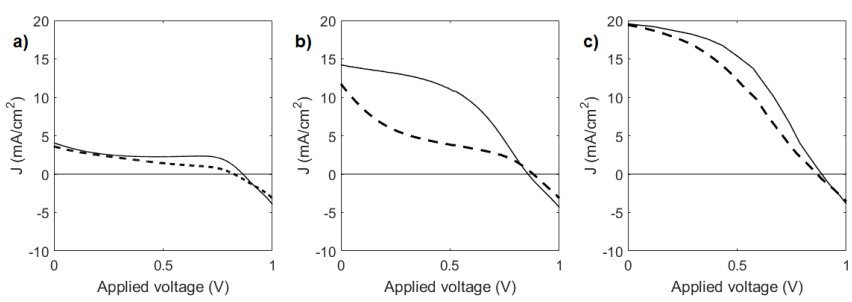

FIG. 2. Experimental JV curves for structure a illustrating normal hysteresis with scan rates of a $1 \mathrm{mV} / \mathrm{s}$, b $75 \mathrm{mV} / \mathrm{s}$ and c $1 \mathrm{~V} / \mathrm{s}$ at $315 \mathrm{~K}$. Solid lines indicate the reverse scan and dashed lines the forward scan.

ments of the temperature dependence of $J_{s c}$ and $H$ vs scan rate for all four cell structures without the need to model $\mathrm{J}$ $\mathrm{V}$ curves for these devices. These $E_{A}$ values are compared with our DFT predictions. In addition, we provide predictions from our model for the sensitivity of PCE vs scan rate curves to $N_{0}$, recombination rates and $E_{A}$. Our concluding section summarises our results and discusses the implications of ion motion for cell stability.

\section{RESULTS AND DISCUSSION}

All parameters for the simulation, including the electron and hole recombination velocities at the interfaces between the perovskite layer and charge transport layers, are stated in Table II of the Supplementary Material, SM, with their sources.

To demonstrate our argument that $E_{A}$ can be obtained directly from experiment, the cells we measured were chosen to be those with the most hysteresis (rather than cells with the highest attainable PCEs). In these cells, bulk recombination dominates so PCEs are around $12 \%$. These cells were chosen to provide the best possible validation of our approach to determine $E_{A}$. Their low PCEs do not limit the validity of our model, which is applicable to a cell regardless of its PCE, nor do they affect the conclusions drawn here. Figure 2a-c shows measured J-V curves at scan rates (a) slow ( $1 \mathrm{mV} / \mathrm{s})$, (b) intermediate $(75 \mathrm{mV} / \mathrm{s})$ and $(\mathrm{c})$ fast $(1 \mathrm{~V} / \mathrm{s})$. The applied bias starts at $1.05 \mathrm{~V}$ decreasing to $0 \mathrm{~V}$ in the reverse scan followed immediately by the forward scan where it is increased to $1.05 \mathrm{~V}$. Predicted J-V curves are shown in Figure 3a-c for scan rates chosen to line up with variations in $H$ : (a) slow $(1 \mathrm{mV} / \mathrm{s})$, (b) intermediate $(320 \mathrm{mV} / \mathrm{s})$ and $(\mathrm{c})$ very fast $(100 \mathrm{~V} / \mathrm{s})$. The simulated characteristic in Figure 3b shows that at intermediate scan rates, the forward scan current density $J$ drops rapidly. This drop is responsible for the difference in the values of $J_{s c}$, 
a)
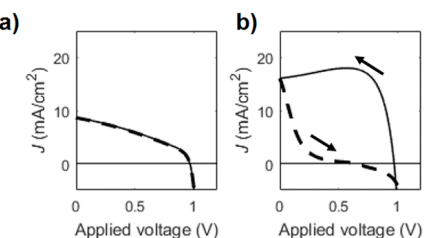

d)

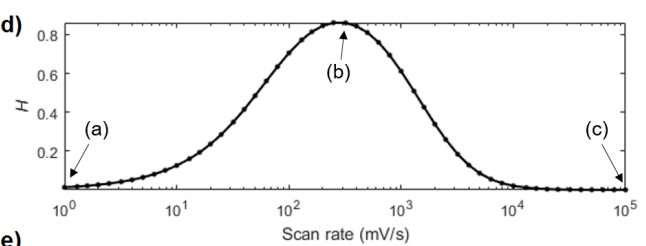

e)

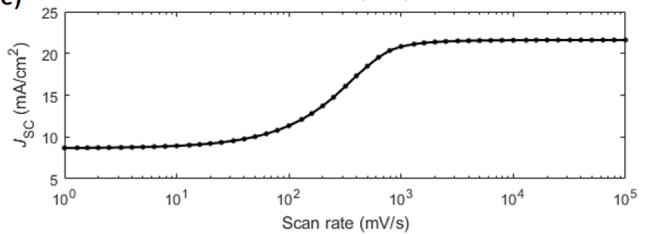

FIG. 3. Panels a,b,c: simulated J-V curves at $315 \mathrm{~K}$ for structure a with scan rates indicated by arrows in panel $\mathrm{d}$ : (a) $1 \mathrm{mV} / \mathrm{s}$, (b) $320 \mathrm{mV} / \mathrm{s}$ and (c) $100 \mathrm{~V} / \mathrm{s}$. The parameters used for these simulations are given in Table $\mathrm{S} 2$ of the $\mathrm{SM}$ including $E_{A}$ set at $0.58 \mathrm{eV}$ for a cubic structure. Solid lines indicate the reverse scan and dashed lines the forward scan. Model predictions for fixed ions are the same as those for the $100 \mathrm{~V} / \mathrm{s}$ scan in panel c; without moving ions there is no dependence on scan rate, and no hysteresis. Panel d: Hysteresis factor $H$ as a function of scan rate. Panel e: Predicted $J_{s c}$ as a function of scan rate.

of around $2 \mathrm{~mA} / \mathrm{cm}^{2}$, observed between the reverse and forward scans in Figure 2b. The difference in these values arises because the potentiostat is digitised with a minimum step size of $10 \mathrm{mV}$ which forces the the scan direction to be reversed at $-8 \mathrm{mV}$ rather than $0 \mathrm{~V}$. This difference does not affect the conclusions drawn here. Figure 3d,e are plots of $H$ and $J_{s c}$ respectively vs scan rate for scan rates from $1 \mathrm{mV} / \mathrm{s}$ to $100 \mathrm{~V} / \mathrm{s}$. The arrows in Figure 3d show that at scan rates (a) and (c) $H$ the rate of change of $J_{s c}$ are close to zero, while at scan rate (b) $H$ and the rate of change of $J_{s c}$ are close to their peak values.

To simulate a J-V curve, the device is initially held at a bias equal to the built-in voltage of $1.05 \mathrm{~V}$ where the internal field is zero, for 5 minutes under illumination. Therefore vacancies are uniformly distributed at the start of the reverse scan. Figures 2a (measurement) and 3a,d,e (simulation) demonstrate that little hysteresis is seen at slow scan rates. Here ion motion is sufficiently fast compared to changes in applied voltage, that the ions keep up with the voltage changes as the applied bias is scanned. The cell behaves as though it were at steadystate at the given applied voltage. In this state, the Debye layers at the perovskite/transport layer interfaces screen the electric field from the central region of the perovskite layer, inhibiting migration of electrons and holes to their respective transport layers. Consequently, the bulk recombination rate within the perovskite is increased above the level and the current density reduced compared to their values if no mobile ions were present. Discrepancies between Figure 2a and Figure 3a may arise from device memory from the previous scans due to very slow moving ion species ${ }^{14}$ and from degradation; a $1 \mathrm{mV} / \mathrm{s}$ scan rate, for example, measuring a full scan from $1.2 \mathrm{~V}$ requires the device to be continuously operated for around 40 minutes.

Hysteresis is seen at intermediate scan rates in Figures $2 b$ and $3 \mathrm{~b}$,d,e. As the applied voltage is increased from zero in the forward scan, the current drops steeply initially then flattens out before eventually dropping below zero. Quantitative differences between measured and simulated $\mathrm{J}-\mathrm{V}$ curves arise from the sensitivity of the simulations to bulk and surface recombination rates. These rates and the relative importance of bulk compared to surface recombination depend on the processing protocols ${ }^{33}$.

For very fast scans shown in Figures 2c and Figure 3c,d,e, the ions do not have sufficient time to move and so are effectively fixed in their initial positions. The current is therefore independent of the scan direction given that electron and hole motion is always much faster than vacancy motion. This prediction is shown in supplementary video 3. Comparing Figure 2c and Figure 3c, there is qualitative agreement between experiment and simulation, indicating that charge carrier transport is accurately modelled in the absence of hysteresis.

A more detailed understanding of how the interaction between ionic and electronic transport influences device characteristics is obtained from supplementary videos $1,2,3$ that show the evolution of the potential profiles when the ion vacancies to move towards a new equilibrium following the applied voltage during the scan at the rates used in panels a-c in Figure 3 respectively. Panels in these videos simulated ion vacancy density, band profiles, current density and bulk electric field over the course of J-V scans. Stills from supplementary video 2 are shown in the supplementary material, SM. These videos are listed at the end of this paper.

Our predicted potential profiles show qualitative agreement with measured profiles from Kelvin probe force microscopy in Figure 3 of reference ${ }^{34}$. We can draw the following conclusions from the videos. Firstly, during a scan, the magnitude and sign of the electric field across the bulk of the perovskite layer are affected by changes in both the applied voltage and the potential drops across each Debye layer. The latter lag behind the scan so the internal electric field within the device depends not only on the applied voltage, but also on the scanning history. Secondly, as seen in the stills included in SM Figure S1, the bulk field changes from positive to negative between reverse and forward scans. When the field is positive, elec- 
trons and holes tend to drift towards their respective transport layers, leading to reduced recombination and a higher current density on the reverse scan. Conversely, a negative bulk field by driving carriers in the opposite direction, increases recombination near the interfaces and decreases the cell current density. Thirdly, close to the interfaces there are strong electric fields that enhance the extraction of charge carriers within a few Debye lengths of the interfaces with the transport layers and so reduce interfacial recombination ${ }^{10}$. These fields affect free carrier extraction rates since these rates depend on the difference between the flux leaving the perovskite layer (determined by the gradient of the local charge quasi-Fermi levels) and the surface recombination flux (that varies as the product of the surface recombination velocities and the electron and hole densities at the interface)

TABLE I. Measured activation energies $E_{A}$ for each device, using the changes in short-circuit current density $\Delta J_{s c}$ and hysteresis factor $\Delta H$ with temperature shown in Figure 5. Use of a spiro-OMeTAD HTL and a Au back contact were common to all device architectures. DFT values are for tetragonal MAPI, FAPI and MAPBr.

\begin{tabular}{lllll}
\hline \hline Perovskite & $\mathrm{ETL}$ & $E_{A}\left(\Delta J_{s c}\right)(\mathrm{eV})$ & $E_{A}(\Delta H)(\mathrm{eV})$ & $E_{A}(\mathrm{DFT})(\mathrm{eV})$ \\
MAPI & $\mathrm{TiO}_{2}$ & 0.41 & 0.45 & 0.44 \\
MAPI & $\mathrm{SnO}_{2}$ & 0.43 & 0.28 & 0.44 \\
FAPI & $\mathrm{SnO}_{2}$ & 0.42 & 0.55 & 0.45 \\
MAPBr & $\mathrm{SnO}_{2}$ & 0.22 & 0.28 & 0.36 \\
\hline \hline
\end{tabular}

Figures $4 \mathrm{a}$ and $\mathrm{b}$ show simulated $H$ and $J_{s c} / J_{s c m a x}$, respectively, where $J_{\text {scmax }}$ is the limit as the scan rate tends to infinity, as a function of scan rate for structure a at temperatures $T$ from $270 \mathrm{~K}$ to $310 \mathrm{~K}$. These curves identify scan rates that measure of the speed of the mobile vacancy response. The peak in $H$ and onset of current loss are shifted down to the $100 \mathrm{mV} / \mathrm{s}$ regime upon cooling. The same effect has been observed in MAPI cells employing organic transport layers ${ }^{35,36}$. For the sigmoidal $J_{s c}$ vs scan rate curves in Figure $4 \mathrm{~b}$, we choose the scan rate giving $90 \% J_{\text {scmax }}$ since this scan rate lies below the large range of scan rates at which $J_{s c}$ is close to $J_{\text {scmax }}$. For $H$, we choose the scan rate at which $H$ obtains its maximum. Figure 4 yields $E_{A}$ from these characteristic scan rates plotted vs $1 /\left(k_{B} T\right)$, from which $E_{A}$ is deduced given the Arrhenius temperature dependence of $D_{v}$. Figure 5 panels a-d show the Arrhenius plots used to extract the values of $E_{A}$ from experiment, for each of our four structures shown on the left of each panel. Purple circles indicate the scan rates at which $J_{s c}$ is $90 \%$ of $J_{\text {scmax }}$ (for reasons stated in the discussion on Figure 4) and green squares the scan rates at which $H$ reaches its maximum, vs $1 /\left(k_{B} \mathrm{~T}\right)$. The measurements of $H$ and $J_{s c}$ versus scan rate as the temperature $T$ varies from which these plots are taken are shown in Figure S2 of the SM. For our MAPI and FAPI devices, hysteresis was still measurable at scan rates down to $1 \mathrm{mV} / \mathrm{s}$ making the limit at low scan rates where $H$ tends to zero hard to ascertain. We suggest the use of settled measurements, for example, holding the device at maximum power point for PCE or holding at open-circuit for $V_{o c}$, so that the property of interest relaxes to its stable value. Table I compares our $E_{A}$ values deduced from measurements of $H$ and
$J_{S c}$ versus scan rate as the temperature $T$ varies for structures a-d and shown in Figure 5 with predicted $E_{A}$ for halide motion for each of the three perovskite materials used in the absorber layers using DFT methods for tetragonal MAPI, FAPI and MAPBr. For MAPBr, Oranskaia et al ${ }^{37}$ predict formation energies of $\mathrm{Br}$ vacancies in MAPBr and FAPBr of $1.25 \mathrm{eV}$ and $1.28 \mathrm{eV}$ respectively and $E_{A}$ of $0.27 \mathrm{eV}$ and $0.33 \mathrm{eV}$ respectively. Our values for $E_{A}$ deduced from $J_{s c}$ measurements are within $10 \%$ of our DFT prediction of $0.44 \mathrm{eV}$ for tetragonal MAPI and to $0.43 \mathrm{eV}$ deduced from measurements of bulk conductivities ${ }^{38}$. $E_{A}$ predicted by first-principles calculations can vary from 0.06 to $0.65 \mathrm{eV}$, depending on the MA orientation $^{21}$. For the MAPBr device, structure d, the predicted $E_{A}$ from experiment disagrees with the DFT calculations by $40 \%$. This discrepancy may be linked either to the lower temperatures where hysteresis occurs due to the lower $E_{A}$ compared to MAPI, or to inaccuracies in the DFT calculations for MAPBr. From the comparisons with DFT values in Table I, measuring $J_{s c}$ gives a more reliable measure of $E_{A}$ than measuring $H$. The maximum in $H$ vs scan rate depends on the shape of the entire $\mathrm{J}-\mathrm{V}$ curve which is sensitive to a wide variety of parameters and interpolating to find the maximum introduces additional error. In contrast, finding the scan rate at which $J_{s c}$ is $90 \%$ of $J_{s c m a x}$ depends on one point on the $\mathrm{J}-\mathrm{V}$ curve, does not include any effects from the change in scan direction and is calculated from monotonic plots of $J_{s c}$ against scan rate. In Figure 6 the effects of ionic motion and carrier recombination on the apparent PCE, defined as the maximum value of $J V$ on the $J-V$ curve obtained by scanning from just above $V_{o c}$ (i.e. $1.2 \mathrm{~V}$ ) to $J_{s c}$ at a fixed scan rate, is shown as a function of the scan rate. Part of the point of this plot is to indicate that there are some quantities, such as defect activation energy $E_{A}$, for which certain changes (e.g. increasing $E_{A}$ ) give rise to measurements that may lead to the mistaken impression that they improve stabilised PCE, whereas in fact the results of these changes lead only to a slowing of the ion dynamics. Nevertheless changes in other quantities, such as reducting defect density $N_{0}$, can lead to significant improvements in stabilised PCE, but nevertheless using a dynamic measurement to assess this improvement may still lead to significant error. In particular,Figure 6a shows the apparent $\mathrm{PCE}$ as a function of scan rate for the simulated device as the defect density is reduced from $1.6 \times 10^{19} \mathrm{~cm}^{-3}$ by three orders of magnitude. We see that the hysteresis and loss behaviours are shifted to longer timescales and reduced in severity. Our explanation is as follows. As the total number of mobile defects decreases, the potential changes from the Debye regions in the perovskite layers increase at the expense of the potential changes linked to the Debye regions in the transport layers. In addition, the Debye layer widths increase. The ratio of the total potential drop across an interfacial Debye layer in the perovskite to the potential drop in the adjacent transport layer is determined by the dimensionless ratio between the product of permittivity and vacancy density in the perovskite and the product of permittivity and doping density in the adjacent transport layer ${ }^{10}$. For the ETL/perovskite interface this ratio is denoted by $\Omega_{E}^{2}$ while for the perovskite/HTL interface it is denoted by $\Omega_{H}^{2}$. The effects on cell performance of varying 

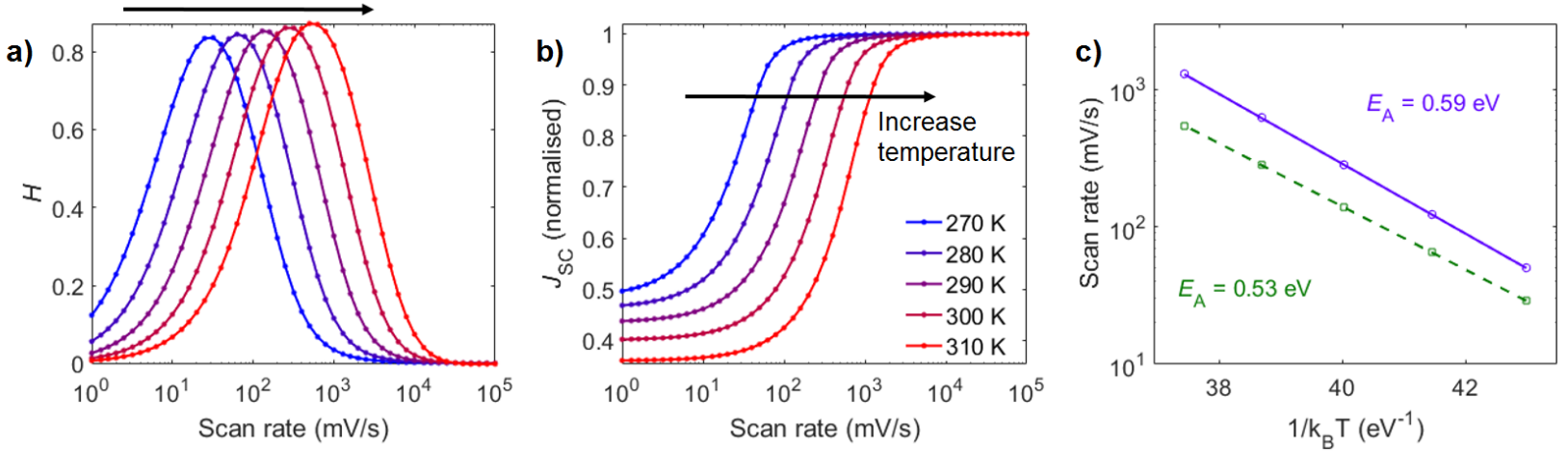

FIG. 4. Panel a Simulated hysteresis factor $H$, panel b ratio of the short-circuit current density $J_{s c}$ to its limiting value $J_{s c m a x}$ vs scan rate for structure a. Simulation parameters are shown in Table II of the SM including $E_{A}$ set at $0.58 \mathrm{eV}$ for a cubic structure. The temperature $T$ runs from $270 \mathrm{~K}$ (blue, leftmost curves) to $310 \mathrm{~K}$ (red, rightmost curves) in steps of $10 \mathrm{~K}$. Panel c Arrhenius plot of the scan rates at which $J_{s c}$ is $90 \% J_{\text {scmax }}$ (purple circles, solid lines giving fit to circles, see text for choice of $90 \%$ ) and where $H$ is a maximum (green squares, dashed lines giving fit to squares). Temperatures run from $330 \mathrm{~K}$ down to $276 \mathrm{~K}$. $E_{A}$ is obtained from the gradient of each line and is shown on the plot.

these two ratios by changing the transport layer properties are discussed in ${ }^{10}$. Our model shows that decreasing either the ion vacancy density in the perovskite or the permittivity in the transport layer, and hence $\Omega_{E}$ and $\Omega_{H}$, leads to proportionally larger potential drops within the perovskite layer that extend further into the perovskite (Supplementary Information Figure 4). In turn, for lower vacancy densities the electric fields close to the perovskite/TL interfaces are larger and extend further into the perovskite layer, giving rise to more efficient carrier extraction to the contacts and reduced recombination losses.

Where the vacancy density decreases below $10^{17} \mathrm{~cm}^{-3}$, the Debye length becomes so large that the Debye layers extend across the entire cell. As a consequence the positive electric field on the reverse scan spreads across the entire width of the perovskite layer, even in the slow scan limit, leading to efficient charge separation throughout the perovskite. As shown in Figure 6a, the apparent PCE at low defect densities represented by the blue line at all scan rates is close to the apparent PCE for higher defect densities at fast scans during which De- bye layers do not have time to form. Larger potential drops within the perovskite as the defect density is reduced lead to vacancies from deeper within the perovskite being drawn into the Debye layers as the charge within these layers evolves. As a consequence, the response time of the ions to a change in applied bias increases, causing an increase in the timescale at which hysteresis is a maximum which corresponds to the shift to slower scan rates as the vacancy density is reduced seen in Figure 6a.

While the formation of the Debye layers tends to reduce the electric field within the bulk of the absorber, many perovskite devices still show good performance on the forward scan, during which the bulk electric field can be negative, by exhibiting low bulk recombination. Charge carrier diffusion lengths/lifetimes in the perovskite material are often sufficiently long to produce a significant current even where charge extraction takes place via diffusion against an adverse potential gradient. In Figure $\mathbf{6 b}$, we show the dependence of performance on the amount of bulk recombination within the perovskite layer and surface recombination at the inter- 
a)

\begin{tabular}{|c|}
\hline $\mathrm{Au}$ \\
\hline spiro \\
\hline $\mathrm{MAPI}$ \\
\hline $\mathrm{TiO}_{2}$ \\
\hline $\mathrm{FTO}$ \\
\hline
\end{tabular}

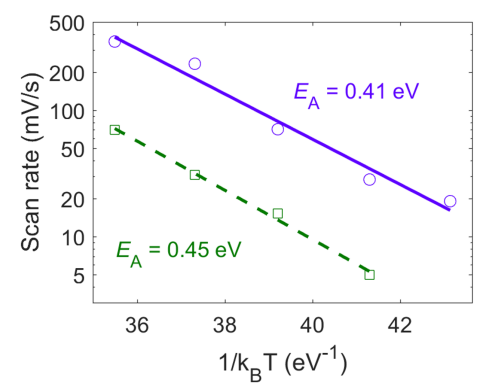

c)

\begin{tabular}{|c|}
\hline $\mathrm{Au}$ \\
\hline spiro \\
\hline FAPI \\
\hline $\mathrm{SnO}_{2}$ \\
\hline FTO \\
\hline
\end{tabular}

b)

\begin{tabular}{|c|}
\hline $\mathrm{Au}$ \\
\hline spiro \\
\hline $\mathrm{MAPI}$ \\
\hline $\mathrm{SnO}_{2}$ \\
\hline $\mathrm{FTO}$ \\
\hline
\end{tabular}

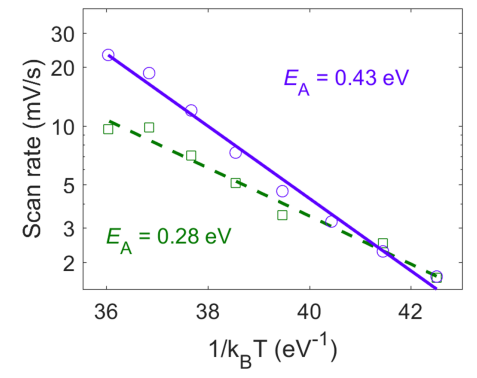

d)
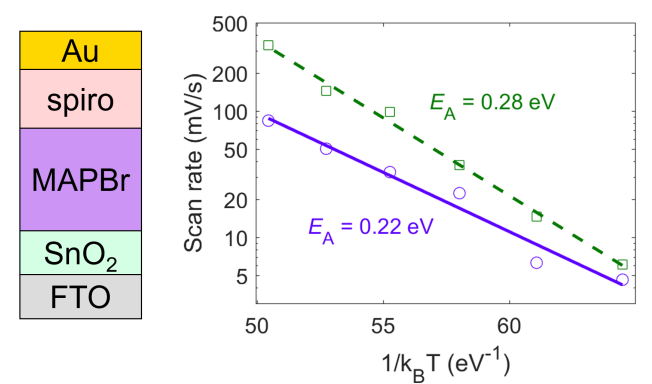

FIG. 5. As for Figure 4 but here each panel shows experimental measurements on each of structures a-d on the left of the panel. $E_{A}$ obtained from the gradient of each line is shown on the plot and listed in Table 1. Panels a-c show results at temperatures of 330K down to $276 \mathrm{~K}$ and panel $\mathrm{d}$ at temperatures of $230 \mathrm{~K}$ down to $276 \mathrm{~K}$.

faces for our simulated structure a. Bulk recombination may be increased by recombination at grain boundaries within the perovskite layer as observed in a recent review by Tennyson et $\mathrm{al}^{39}$. The timescale of ion motion is unaffected by the level of recombination, and so the scan rate dependence of the behaviour displayed in Figure $\mathbf{6 b}$ is largely unchanged. The performance improvement as bulk and surface recombination are reduced for all scan rates is shown in Figure $\mathbf{6 b}$. The greatest improvement in apparent PCE is for slower scans, where losses due to poor charge separation are largest. Supplementary Material Figure 3 explores the variation of the four standard metrics of performance $\left(J_{s c}, V_{o c}\right.$, PCE and FF) with the recombination multiplier defined in the caption for Figure 6.

In Figure 6c, we show the effect of increasing $E_{A}$ from $0.58 \mathrm{eV}^{22}$, to 0.68 and $0.78 \mathrm{eV}$. Debye layers still form regardless of the value of $E_{A}$, albeit more slowly as $E_{A}$ increases, and so there is no resulting increase in the settled PCE, given by the apparent PCE at slow scan rates in Figure 6c.

\section{CONCLUSIONS}

We have investigated transient behaviour exhibited by perovskite solar cells (PSCs) with a mix of experiment and theory. From a drift-diffusion model that accounts for both ion vacancy and charge carrier motion, we can reproduce the observed behaviour of PSCs during $\mathrm{J}-\mathrm{V}$ scans, including the dependence of the J-V curve on scan rate and temperature. Videos in the Supplementary Information show the cell response throughout a $\mathrm{J}-\mathrm{V}$ scan. In line with previous results ${ }^{25,40-43}$, we find that hysteresis in the $\mathrm{J}-\mathrm{V}$ curves of PSCs is primarily caused by the flow of halide ions in and out of narrow Debye layers at the edges of the perovskite film which screen the electric field.

Furthermore, we show that the extent to which hysteresis is present depends on the alignment of the timescales of the scan and ion motion within the film. In the devices we considered, screening of the electric field from the perovskite layer significantly reduces device performance by hindering charge separation in the perovskite, even where hysteresis is not observed. However, for cells fabricated with sufficiently long charge carrier diffusion lengths, where charge separation is 

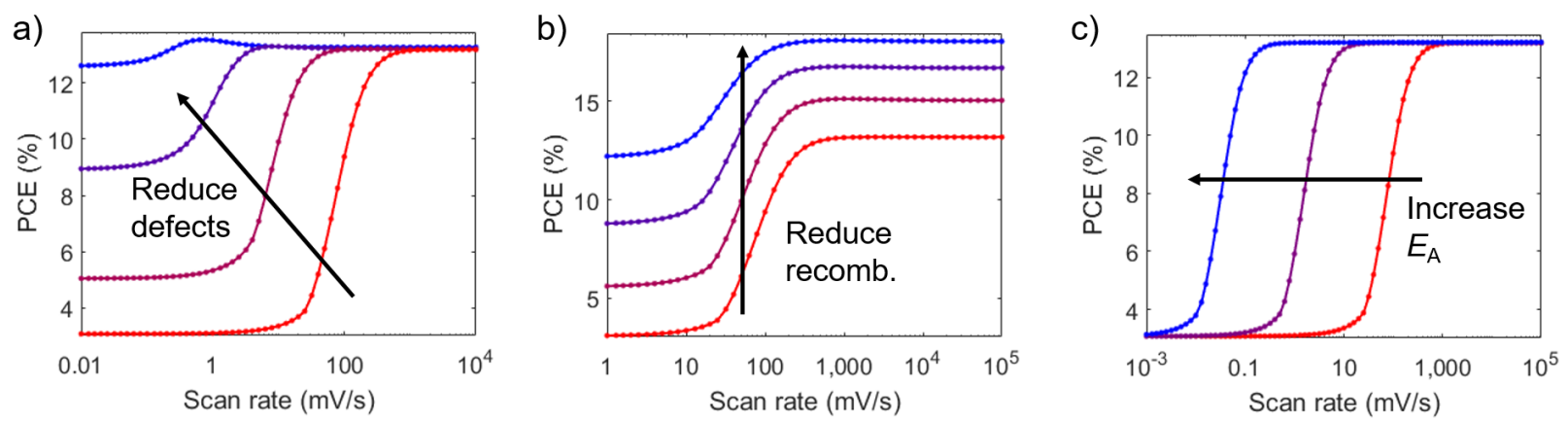

FIG. 6. Predicted apparent power conversion efficiency PCE as a function of scan rate for the device used for Figure 2 Simulation parameters are shown in Table II of the SM and the following parameters are varied. Panel a The defect density $N_{0}$ is reduced from $1.6 \times 10^{19}$ (red) to $1.6 \times 10^{16} \mathrm{~cm}^{-3}$ (blue) in decades. Panel b All recombination parameters are made less severe from their original values (red) to most favourable (blue). The values of $\beta, v_{n E}, v_{p E}, v_{n H}$ and $v_{p H}$ are taken from the values shown in Table S2, divided by 1, 2, 4 and 8 . Values of $\tau_{n}$ and $\tau_{p}$ come from Table S2, multiplied by 1, 2, 4 and 8. Panel c $E_{A}$ for halide motion is increased from 0.58 (red) to 0.68 and $0.78 \mathrm{eV}$ (blue).

not such a major issue, this need not be the case. Moreover, we were able to confirm predictions from the model by comparing to experimental data from the four different PSCs.

If hysteresis is not present when a device is measured, we suggest investigating other scan rates and temperatures to find the slow scan limit. For our MAPI and FAPI devices, hysteresis was still present even for scan rates down to $1 \mathrm{mV} / \mathrm{s}$. To remedy this problem, we suggest the use of settled measurements, for example, holding the device at maximum power point for PCE or holding at open-circuit for $V_{o c}$, so that the property of interest relaxes to its stable value.

Our model is predictive in that we have shown how the short circuit current density and hysteresis factor can be used to predict the energy of ion motion $E_{A}$. We have used these predictions to establish an experimental technique for extracting the activation energies of ion motion in real devices. This technique requires measurements of the temperature variation of: (i) the scan rate corresponding to maximum hysteresis; (ii) the scan rate at which $J_{s c}$ reaches $90 \%$ of its maximum value. The results we obtained from these two sets of measurements are in broad agreement with each other and with DFT calculations and other experimental values.

We find that reductions in ion vacancy density reduce recombination and improve settled PCE for cells limited by recombination in the bulk of the perovskite. In contrast, we found slowing down the motion of ion vacancies by increasing the activation energy shifts losses to longer timescales but does not mitigate efficiency loss at steady state.

A device appearing to be hysteresis-free may be scanned at a rate or a temperature at which hysteresis is negligible. Measurable levels of hysteresis have been seen when the temperature is changed. For example, a MAPI cell with organic transport layers that was hysteresis-free at room temperature and standard scan rates exhibited severe hysteresis upon cooling to $175 \mathrm{~K},{ }^{35}$.

Our model shows that hysteresis can be reduced by lowering $N_{0}$ and recombination rates. This result explains why the following approaches have been found to achieve these goals: (i) widespread adoption of mixed cation perovskites $^{44-46}$; (ii) surface passivation which reduces sur- 
face vacancy densities ${ }^{47}$; (iii) minimisation of compressive lattice strain, to reduce the equilibrium halide vacancy density correlates with strain and that strained regions show increased non-radiative recombination ${ }^{48}$.

The strain caused by ion motion occurs in the regions of significant build-up and depletion of the ion vacancy density at the edges of the perovskite. Ion build-up/depletion has also been found to lead to carrier build-up $25,42,43,49$, which in turn contributes to additional Ohmic heating and thermal breakdown ${ }^{50}$. This heating may cause problems if the temperature within the perovskite passes through a phase transition, for example the transition in MAPI around $327 \mathrm{~K}$ from tetragonal to cubic ${ }^{51}$. Another degradation pathway in perovskites has been linked to the highly reactive $\mathrm{O}_{2}^{-}$superoxide species that is generated from the reaction of free electrons in the perovskite with molecular oxygen. Ab initio simulations indicate that iodide vacancies are the preferred site for $\mathrm{O}_{2}^{-}$formation ${ }^{52}$. Therefore reducing the ion vacancy density is likely to benefit not just cell performance but also the long-term stability of PSCs.

\section{METHODS}

\section{Modelling}

A description of the DFT based calculations for the $E_{A}$ values is given in section 2 of the Supplementary Information.

Our drift-diffusion (DD) picture is described by Courtier et $\mathrm{al}^{10}$. We model the motion of electrons, holes and halide ion vacancies within a planar PSC. The perovskite absorber and both transport layers (TLs) are all explicitly modelled. A similar model has been employed by others ${ }^{53,54}$ to study impedance and transient current measurements. Our model goes beyond earlier work by ourselves ${ }^{25}$ and others ${ }^{41}$ by explicitly including the TLs, allowing for conduction and valence band offsets at the ETL/perovskite and perovskite/HTL interfaces. Inclusion of the TLs is crucial for accurate simulation of recombination at the TL/perovskite interfaces. We go beyond ${ }^{25,55}$ in including the influence of carriers on the potential and beyond ${ }^{56}$ by allowing the distribution of ions to vary during $\mathrm{J}-\mathrm{V}$ scans. The assumed value of the ion diffusion coefficient in reference ${ }^{40}$ is $10^{6}$ times greater than that of reference 22 and voltage scan rates around $10^{6}$ times greater than the experimental scan rates at which hysteresis is observed. $\operatorname{In}^{57}$, the modelled ion concentration profile is fixed to its equilibrium profile.

In some descriptions of ion motion positive and negative species are assumed to have similar mobilities ${ }^{12}$, causing a spatially symmetric distribution of charge with respect to an axis midway across the perovskite layer. We argue that this description is incorrect. Migration of iodide anions via lattice interstitials will not contribute appreciably to the overall ion transport. In Minns et $\mathrm{al}^{58}$, no measurement of the ion diffusion coefficient is reported and the concentration of interstitial species is low. $\mathrm{MA}^{+}$cations have also been suggested to contribute to ion conduction but their small diffusion coefficient rules out a significant contribution at the same timescales as that of the halide vacancies ${ }^{59}$. Wu et al ${ }^{12}$ employed this model to study steady state characteristics but we would note that such a model is only valid for times much longer than the time for ion vacancies to migrate across the perovskite layer and at these long timescales we found experimental evidence of degradation.

We numerically integrate the full drift-diffusion equations using a scheme based on the work of Courtier et $\mathrm{al}^{43}$ that has been used to validate simulations with one-dimensional solver using the Quokka3 software ${ }^{60}$. Courtier et al's scheme makes use of a non-uniform spatial discretisation that clusters points around the ETL/perovskite and perovskite/HTL interfaces where the presence of Debye layers gives rise to extremely rapidly varying solutions. A detailed description of the model, and its numerical solution, is given in section 3 of the Supplementary Information. In particular, the full set of equations is given in (S1)-(S15) with boundary conditions in (S16)-(S31). Table 1 of the Supplementary Information defines the symbols. The applied voltage and built-in field are included via appropriate boundary conditions on Poisson's equation. The time variation of the applied voltage is accomplished by making the boundary conditions a function of time.

Electrons and holes are generated and can recombine in the perovskite. Electrons exist only in the perovskite and ETL while holes exist only in the perovskite and HTL due to the large energy barriers (more than an $\mathrm{eV}$ ) that prevent each type of carrier from entering the opposite TL. The ratio of the carrier density on the higher-energy side of a potential barrier $\Delta E$ compared to on the lower-energy side is given by Boltzmann statistics to be $\exp -\Delta E / k_{B} T$. For a modest barrier of $1 \mathrm{eV}$ at room temperature, this factor gives a reduction in density of around 17 orders of magnitude.

\section{Experiment}

The materials used, their sources and device fabrication are stated in section 1 of the Supplementary Information The J- V response of the perovskite devices was recorded as a function of sweep rate (1000 to $1 \mathrm{mV} / \mathrm{s}$ in logarithmic steps with $\Delta \mathrm{V}$ $=10 \mathrm{mV}$ ) with Metrohm Autolabs PGSTAT12 potentiostat. Devices were pre-conditioned under illumination at $+1.2 \mathrm{~V}$ (or $+1.6 \mathrm{~V}$ for MAPBr) forward bias for $5 \mathrm{~min}$ before each scan. Reverse scans (forward bias to short circuit) were recorded before immediately switching into forward scans (short circuit to forward bias). After each scan, the device would again equilibrate for $5 \mathrm{~min}$ under forward bias before moving onto the next sweep rate.

Illumination was not interrupted until after completion of the $\mathrm{J}-\mathrm{V}$ regimen. Temperature and illumination control were implemented by a Linkam LTS-420-E cryostat connected to a PAIOS 2.0 characterisation system (Fluxim, Switzerland).The cryostage was purged with a protective layer of dry nitrogen before use.Sample temperature was recorded with a PT100 resistance temperature detector in thermal contact with the solar cell. Illumination came from a white LED coupled into the cryostat with a light tube. The cell PCEs were evaluated from $\mathrm{J}-\mathrm{V}$ characteristics obtained at a scan rate of $1 \mathrm{mV} / \mathrm{s}$ at $1 \mathrm{Sun}$ illumination. 


\section{SUPPLEMENTARY MATERIAL}

Section A of the Supplementary Material, SM, covers Materials Synthesis and Device Fabrication, section B DFT simulation, section C the Drift Diffusion model, Figure 1 Stills from Supplementary Video 2, Figure 2 device structures, $H$ and $J_{s c}$ vs scan rate and Arrhenius fits of the measured scan rate, Figure 3 Variation of $H, J_{s c}$ PCE, FF and $V_{o c}$ with.the recombination multiplier $R_{\text {mult }}$, Figure 4 simulated distribution of the electric potential and he normalised ion vacancy distribution across the perovskite.

\section{Supplementary Videos}

These videos show top left electric potential (black) (V), ion density (pink) $\left(\mathrm{cm}^{-3}\right)$ profiles; top right: electric potential (V)/electron density (red), hole density (blue) $\left(\mathrm{cm}^{-3}\right)$ profiles, bottom left current density $\left(\mathrm{mA} / \mathrm{cm}^{2}\right)$ vs applied voltage $(\mathrm{V})$ bottom lright bulk electric field (MV/m) vs applied voltage (V) as the applied bias is scanned from $V_{o c}$ down to zero in a reverse scan followed by a forward scan from zero to $V_{o c}$. Supplementary Video 1: slow scan $(1 \mathrm{mV} / \mathrm{s})$, Supplementary Video 2: medium scan $(320 \mathrm{mV} / \mathrm{s})$, Supplementary Video 3:f ast scan $(100 \mathrm{~V} / \mathrm{s})$.

\section{DATA AVAILABILITY STATEMENT}

The data that supports the findings of this study are available within the article [and its supplementary material]

\section{AUTHOR CONTRIBUTIONS}

JMC, ABW, GR and MSI wrote the manuscript. JMC ran the simulations. JMC, IB, AD and TWJ made experimental measurements. DG carried out DFT simulations. JMC, NEC and JMF developed and coded the DD numerical scheme. JMC, NEC, KF, GR and ABW contributed to interpreting the DD model. JMF and GR conceived and formulated the DD model. TWJ, KA and LL fabricated the devices. KF, JMF, GR, MSI and ABW supervised theoretical work. TWJ and GJW supervised experimental work. All authors contributed to revising the manuscript.

\section{ACKNOWLEDGMENTS}

We would like to thank Chris Fell and Laurie Peter for their careful reading of the manuscript. JMC and NEC acknowledge EPSRC funded studentships from the CDT in New and Sustainable Photovoltaics (EP/L01551X/1). This work has received funding from the Australian Renewable Energy Agency (ARENA) as part of ARENA's Emerging Renewables Program via grant P159394. DG, ABW and SI received funding from the European Union's Horizon 2020 research and innovation programme under EoCoE (grant agreement 676629) and ABW also under EoCoE II (grant agreement 824158).
${ }^{1}$ A. Extance, "The reality behind solar power's next star material," Nature 570, 429 (2019).

${ }^{2}$ D. P. Buemi, "Perovskite solar cells: Hero, villain or just plain fantasy?" Solar Power World (2020).

${ }^{3} \mathrm{M}$. S. Islam, "Ionic transport in $\mathrm{ABO}_{3}$ perovskite oxides: a computer modelling tour," J. Mater. Chem. 10, 1027-1038 (2000).

${ }^{4}$ W. A. Dunlap-Shohl, Y. Zhou, N. P. Padture, and D. B. Mitzi, "Synthetic approaches for halide perovskite thin films," Chem. Revs. 119, 3193-3295 (2019).

${ }^{5}$ S. D. Stranks and H. J. Snaith, "Metal-halide perovskite for photovoltaic and light-emitting devices," Nat. Nanotech. 10, 391-402 (2015).

${ }^{6}$ C. Li, S. Tscheuschner, F. Paulus, P. E. Hopkinson, J. Kießling, A. Köhler, Y. Vaynzof, and S. Huettner, "Iodine migration and its effect on hysteresis in perovskite solar cells," Adv. Mater. 28, 2446-2454 (2016).

${ }^{7}$ A. Bercegol, S. Cacovich, G. Vidon, S. Mejaouri, A. Yaiche, J.-B. Puel, C. Longeaud, J.-F. Guillemoles, S. Jutteau, J. Rousset, D. Ory, and L. Lombez, "Imaging electron, hole, and ion transport in halide perovskite," The Journal of Physical Chemistry C 124, 11741-11748 (2020).

${ }^{8}$ W. Yu, F. Li, L. Yu, M. R. Niazi, Y. Zou, D. Corzo, A. Basu, C. Ma, S. Dey, M. L. Tietze, U. Buttner, X. Wang, Z. Wang, M. N. Hedhili, C. Guo, T. Wu, and A. Amassian, "Native defect-induced hysteresis behavior in organolead iodide perovskite solar cells," Nat. Commun. 9, 5354 (2018).

${ }^{9}$ B. Hwang and J.-S. Lee, "Hybrid organic-inorganic perovskite memory with long-term stability in air," Sci. Rep. 7, 673 (2017).

${ }^{10}$ N. E. Courtier, J. M. Cave, J. M. Foster, A. B. Walker, and G. Richardson, "How transport layer properties affect perovskite solar cell performance: insights from a coupled charge transport/ion migration model," Energy Environ. Sci. 12, 396-409 (2019).

${ }^{11}$ M. V. Khenkin, E. A. Katz, A. Abate, G. Bardizza, and J. J. e. a. Berry, "Consensus statement for stability assessment and reporting for perovskite photovoltaics based on isos procedures," Nature Energy 5, 35-49 (2020).

${ }^{12} \mathrm{~N}$. Wu, D. Walter, A. Fell, Y. Wu, and K. Weber, "The impact of mobile ions on the steady-state performance of perovskite solar cells," The Journal of Physical Chemistry C 124, 219-229 (2020).

${ }^{13}$ C.-J. Tong, L. Li, L.-M. Liu, and O. V. Prezhdo, "Synergy between ion migration and charge carrier recombination in metal-halide perovskites," $\mathrm{J}$. Am. Chem. Soc. 142, 3060-3068 (2020).

${ }^{14}$ K. Domanski, B. Roose, T. Matsui, M. Saliba, S.-H. Turren-Cruz, J.-P. Correa-Baena, C. R. Carmona, G. Richardson, J. M. Foster, F. De Angelis, J. M. Ball, A. Petrozza, N. Mine, M. K. Nazeeruddin, W. Tress, M. Grätzel, U. Steiner, A. Hagfeldt, and A. Abate, "Migration of cations induces reversible performance losses over day/night cycling in perovskite solar cells," Energy Environ. Sci. 10, 604-613 (2017).

${ }^{15}$ G. A. Nemnes, C. Besleaga, V. Stancu, D. E. Dogaru, L. N. Leonat, L. Pin tilie, K. Torfason, M. Ilkov, A. Manolescu, and I. Pintilie, "Normal and inverted hysteresis in perovskite solar cells," J. Phys. Chem. C 121, 1120711214 (2017)

${ }^{16}$ G. A. Nemnes, C. Besleaga, A. G. Tomulescu, A. Palici, L. Pintilie, A. Manolescu, and I. Pintilie, "How measurement protocols influence the dynamic j-v characteristics of perovskite solar cells: Theory and experiment," Solar Energy 173, 976 - 983 (2018).

${ }^{17}$ Y. Hou, S. Scheiner, X. Tang, N. Gasparini, M. Richter, N. Li, P. Schweizer, S. Chen, H. Chen, C. O. R. Quiroz, X. Du, G. J. Matt, A. Osvet, E. Spiecker, R. H. Fink, A. Hirsch, M. Halik, and C. J. Brabec, "Suppression of hysteresis effects in organohalide perovskite solar cells," Adv. Mater. Interfaces 4, 1700007 (2017)

${ }^{18}$ H. J. Snaith, A. Abate, J. M. Ball, G. E. Eperon, T. Leijtens, N. K. Noel S. D. Stranks, J. T.-W. Wang, K. Wojciechowski, and W. Zhang, "Anomalous hysteresis in perovskite solar cells," J. Phys. Chem. Lett. 5, 1511-1515 (2014).

${ }^{19}$ S. N. Habisreutinger, N. K. Noel, and H. J. Snaith, "Hysteresis index: A figure without merit for quantifying hysteresis in perovskite solar cells," ACS Energy Letters 118, 2472-2476 (2018).

${ }^{20}$ A. Walsh, D. O. Scanlon, S. Chen, X. G. Gong, and S.-H. Wei, "Selfregulation mechanism for charged point defects in hybrid halide perovskites," Angew. Chem. Int. Ed. 127, 1811 (2015).

${ }^{21}$ C.-J. Tong, W. Geng, O. V. Prezhdo, and L.-M. Liu, "Role of methylammonium orientation in ion diffusion and current-voltage hysteresis in the ch3nh3pbi3 perovskite," ACS En. Letts. 2, 1997-2004 (2017). 
${ }^{22}$ C. Eames, J. M. Frost, P. R. F. Barnes, B. C. O’Regan, A. Walsh, and M. S Islam, "Ionic transport in hybrid lead iodide perovskite solar cells," Nat. Commun. 6, 7497 (2015)

${ }^{23}$ A. Walsh and S. D. Stranks, "Taking control of ion transport in halide perovskite solar cells," ACS Energy Lett. 0, 1983-1990 (2018).

${ }^{24}$ S. Reichert, Q. An, Y.-W. Woo, A. Walsh, Y. Vaynzof, and D. Carsten, "Probing the ionic defect landscape in halide perovskite solar cells," arXiv:2005.06942 (2020)

${ }^{25}$ G. Richardson, S. E. J. O'Kane, R. G. Niemann, T. A. Peltola, J. M. Foster, P. J. Cameron, and A. B. Walker, "Can slow-moving ions explain hysteresis in the current-voltage curves of perovskite solar cells?" Energy Environ. Sci. 9, 1476-1485 (2016)

${ }^{26}$ R. A. Kerner and B. P. Rand, "Ionic-electronic ambipolar transport in metal halide perovskites: Can electronic conductivity limit ionic diffusion?" J Phys. Chem. Lett. 9, 132-137 (2018).

${ }^{27}$ J. Mizusaki, K. Arai, and K. Fueki, "Ionic conduction of the perovskitetype halides," Solid State Ion. 11, 203-211 (1983).

${ }^{28}$ A. Pockett, G. E. Eperon, N. Sakai, H. J. Snaith, L. M. Peter, and P. J. Cameron, "Microseconds, milliseconds and seconds: deconvoluting the dynamic behaviour of planar perovskite solar cells," Phys. Chem. Chem. Phys. 19, 5959-5970 (2017)

${ }^{29}$ A. Rizzo, F. Lamberti, M. Buonomo, N. Wrachien, L. Torto, N. Lago, S. Sansoni, R. Pilot, M. Prato, N. Michieli, M. Meneghetti, G. Meneghesso, and A. Cester, "Understanding lead iodide perovskite hysteresis and degradation causes by extensive electrical characterization," Sol. Energy Mater. Sol. Cells 189, 43 - 52 (2019).

${ }^{30}$ S. Meloni, T. Moehl, W. Tress, M. Franckevičius, M. Saliba, Y. H. Lee, P. Gao, M. K. Nazeeruddin, S. M. Zakeeruddin, U. Rothlisberger, and $\mathrm{M}$. Grätzel, "Ionic polarization-induced current-voltage hysteresis in $\mathrm{CH}_{3} \mathrm{NH}_{3} \mathrm{PbX}_{3}$ perovskite solar cells," Nat. Commun. 7, 10334 (2016).

${ }^{31}$ O. Almora, I. Zarazua, I. Mas-Marza, E.and Mora-Sero, J. Bisquert, and G. Garcia-Belmonte, "Capacitive dark currents, hysteresis, and electrode polarization in lead halide perovskite solar cells," J. Phys. Chem. Lett. 6 , 1645-1652 (2015).

${ }^{32}$ N. E. Courtier, J. M. Foster, S. E. J. O'Kane, A. B. Walker, and R. G., "Ionmonger: a free and fast planar perovskite solar cell simulator with coupled ion vacancy and charge carrier dynamics," Journal of Computational Electronics 18, 1435-1449 (2019).

${ }^{33}$ L. M. Herz, "Charge-carrier dynamics in organic-inorganic metal halide perovskites," Annu. Rev. Phys. Chem. 67, 65-89 (2016).

${ }^{34}$ S. Weber, I. M. Hermes, S. H. Turren Cruz, C. Gort, V. W. Bergmann, L. Gilson, A. Hagfeldt, M. Grätzel, W. Tress, and R. Berger, "How the formation of interfacial charge causes hysteresis in perovskite solar cells," Energy Environ. Sci. , 2404-2414 (2018).

${ }^{35}$ D. Bryant, S. Wheeler, B. C. O'Regan, T. Watson, P. R. F. Barnes, D. Worsley, and J. Durrant, "Observable hysteresis at low temperature in "hysteresis free" organic-inorganic lead halide perovskite solar cells," J. Phys Chem. Lett. 6, 3190-3194 (2015)

${ }^{36}$ I. Levine, P. K. Nayak, J. T.-W. Wang, N. Sakai, S. Van Reenen, T. M. Brenner, S. Mukhopadhyay, H. J. Snaith, G. Hodes, and D. Cahen, "Interfacedependent ion migration/accumulation controls hysteresis in $\mathrm{MAPbI}_{3}$ solar cells," J. Phys. Chem. C 120, 16399-16411 (2016).

${ }^{37}$ A. Oranskaia, J. Yin, O. M. Bakr, J.-L. Brédas, and O. F. Mohammed, "Halogen migration in hybrid perovskites: The organic cation matters," J. Phys. Chem. Lett. 9, 5474 (2018).

${ }^{38}$ T.-Y. Yang, G. Gregori, N. Pellet, M. Grätzel, and J. Maier, "The significance of ion conduction in a hybrid organic-inorganic lead-iodide-based perovskite photosensitizer," Angew. Chem. Int. Ed. 54, 7905 (2015).

${ }^{39}$ E. M. Tennyson, T. A. S. Doherty, and S. D. Stranks, "Heterogeneity at multiple length scales in halide perovskite semiconductors," Nature Reviews Materials , 573-587 (2019).

${ }^{40}$ S. van Reenen, M. Kemerink, and H. J. Snaith, "Modeling anomalous hysteresis in perovskite solar cells," J. Phys. Chem. Lett. 6, 3808-3814 (2015) ${ }^{41}$ D. Moia, I. Gelmetti, P. Calado, W. Fisher, M. Stringer, O. Game, Y. Hu, P. Docampo, D. Lidzey, E. Palomares, J. Nelson, and P. R. F. Barnes, "Ionic-to-electronic current amplification in hybrid perovskite solar cells: ionically gated transistor-interface circuit model explains hysteresis and impedance of mixed conducting devices," Energy Environ. Sci. 12, 12961308 (2019)
${ }^{42}$ S. E. J. O'Kane, G. Richardson, A. Pockett, R. G. Niemann, J. M. Cave, N. Sakai, G. E. Eperon, H. J. Snaith, J. M. Foster, P. J. Cameron, and A. B. Walker, "Measurement and modelling of dark current decay transients in perovskite solar cells," J. Mater. Chem. C 5, 452-462 (2017).

${ }^{43}$ N. E. Courtier, G. Richardson, and J. M. Foster, "A fast and robust numerical scheme for solving models of charge carrier transport and ion vacancy motion in perovskite solar cells," Appl. Math. Model. 63, 329-348 (2018).

${ }^{44}$ D. Ghosh, P. Walsh Atkins, M. S. Islam, A. B. Walker, and C. Eames, "Good vibrations: Locking of octahedral tilting in mixed-cation iodide perovskites for solar cells," ACS Energy Lett. 2, 2424-2429 (2017).

${ }^{45}$ B. Charles, J. Dillon, O. J. Weber, M. S. Islam, and M. T. Weller, "Understanding the stability of mixed A-cation lead iodide perovskites," J. Mater. Chem. A 5, 22495-22499 (2017).

${ }^{46}$ M. Saliba, T. Matsui, J.-Y. Seo, K. Domanski, J.-P. Correa-Baena, M. K Nazeeruddin, S. M. Zakeeruddin, W. Tress, A. Abate, A. Hagfeldt, and M. Grätzel, "Cesium-containing triple cation perovskite solar cells: improved stability, reproducibility and high efficiency," Energy Environ. Sci. 9, 1989-1997 (2016).

${ }^{47}$ S. P. Senanayak, B. Yang, T. H. Thomas, N. Giesbrecht, W. Huang, E. Gann, B. Nair, K. Goedel, S. Guha, X. Moya, C. R. McNeill, P. Docampo, A. Sadhanala, R. H. Friend, and H. Sirringhaus, "Understanding charge transport in lead iodide perovskite thin-film field-effect transistors," Sci. Adv. 3 (2017).

${ }^{48}$ T. W. Jones, A. Osherov, M. Alsari, M. Sponseller, B. C. Duck, Y.-K Jung, C. Settens, F. Niroui, R. Brenes, C. V. Stan, Y. Li, M. Abdi-Jalebi, N. Tamura, J. E. Macdonald, M. Burghammer, R. H. Friend, V. Bulović, A. Walsh, G. J. Wilson, S. Lilliu, and S. D. Stranks, "Lattice strain cause non-radiative losses in halide perovskites," Energy Environ. Sci. 12, 596606 (2019).

${ }^{49}$ P. Calado, A. M. Telford, D. Bryant, X. Li, J. Nelson, B. C. O'Regan, and P. R. Barnes, "Evidence for ion migration in hybrid perovskite solar cells with minimal hysteresis," Nat. Commun. 7 (2016).

${ }^{50} \mathrm{~J}$. O'Dwyer, The theory of electrical conduction and breakdown in solid dielectrics (Clarendon Press, Oxford, 1973).

${ }^{51}$ D. W. Miller, G. E. Eperon, E. T. Roe, C. W. Warren, H. J. Snaith, and M. C. Lonergan, "Defect states in perovskite solar cells associated with hysteresis and performance," Appl. Phys. Lett. 109, 153902 (2016).

${ }^{52}$ N. Aristidou, C. Eames, I. Sanchez-Molina, X. Bu, J. Kosco, M. Islam, and S. Haque, "Fast oxygen diffusion and iodide defects mediate oxygeninduced degradation of perovskite solar cells," Nat. Commun. 8, 15215 (2017).

${ }^{53}$ D. A. Jacobs, H. Shen, F. Pfeffer, J. Peng, T. P. White, F. J. Beck, and K. R Catchpole, "The two faces of capacitance: New interpretations for electrical impedance measurements of perovskite solar cells and their relation to hysteresis," J. Appl. Phys. 124, 225702 (2018).

${ }^{54}$ M. Neukom, A. Schiller, S. Züfle, E. Knapp, J. Ávila, D. Pérez-del Rey, C. Dreessen, K. P. Zanoni, M. Sessolo, H. J. Bolink, and B. Ruhstaller, "Consistent device simulation model describing perovskite solar cells in steady-state, transient, and frequency domain," ACS Appl. Mater. Interfaces 11, 23320-23328 (2019)

${ }^{55}$ T.-Y. Zhu and D.-J. Shu, "Role of ionic charge accumulation in perovskite solar cell: Carrier transfer in bulk and extraction at interface,” The Journal of Physical Chemistry C 123, 5312-5320 (2019).

${ }^{56}$ D. A. Jacobs, Y. Wu, H. Shen, C. Barugkin, F. J. Beck, T. P. White, K. Weber, and K. R. Catchpole, "Hysteresis phenomena in perovskite solar cells: the many and varied effects of ionic accumulation," Phys. Chem. Chem. Phys. 19, 3094-3103 (2017).

${ }^{57}$ L. Bertoluzzi, R. A. Belisle, K. A. Bush, R. Cheacharoen, M. D. McGehee, and B. C. O'Regan, "In situ measurement of electric-field screening in hysteresis-free PTAA/ $\mathrm{FA}_{0.83} \mathrm{Cs}_{0.17} \mathrm{~Pb}\left(\mathrm{I}_{0.83} \mathrm{Br}_{0.17)} 3 / \mathrm{C}_{60}\right.$ perovskite solar cells gives an ion mobility of $3 \times 10^{-7} \mathrm{~cm}^{2} /(\mathrm{Vs}), 2$ orders of magnitude faster than reported for metal-oxide-contacted perovskite cells with hysteresis," JACS 140, 12775-12784 (2018).

${ }^{58}$ J. L. Minns, P. Zajdel, D. Chernyshov, W. van Beek, and M. A. Green, "Structure and interstitial iodide migration in hybrid perovskite methylammonium lead iodide," Nat. Commun. 8, 15152 EP (2017).

${ }^{59}$ A. Senocrate, I. Moudrakovski, G. Y. Kim, T.-Y. Yang, G. Gregori, M. Grätzel, and J. Maier, "The nature of ion conduction in methylammonium lead iodide: A multimethod approach,” Angew. Chem. Int. Ed. 56, 7755-7759 (2017) 
${ }^{60}$ D. Walter, A. Fell, Y. Wu, T. Duong, C. Barugkin, N. Wu, T. White, and

$\mathrm{K}$. Weber, "Transient photovoltage in perovskite solar cells: Interaction of trap-mediated recombination and migration of multiple ionic species," The
Journal of Physical Chemistry C 122, 11270-11281 (2018). 


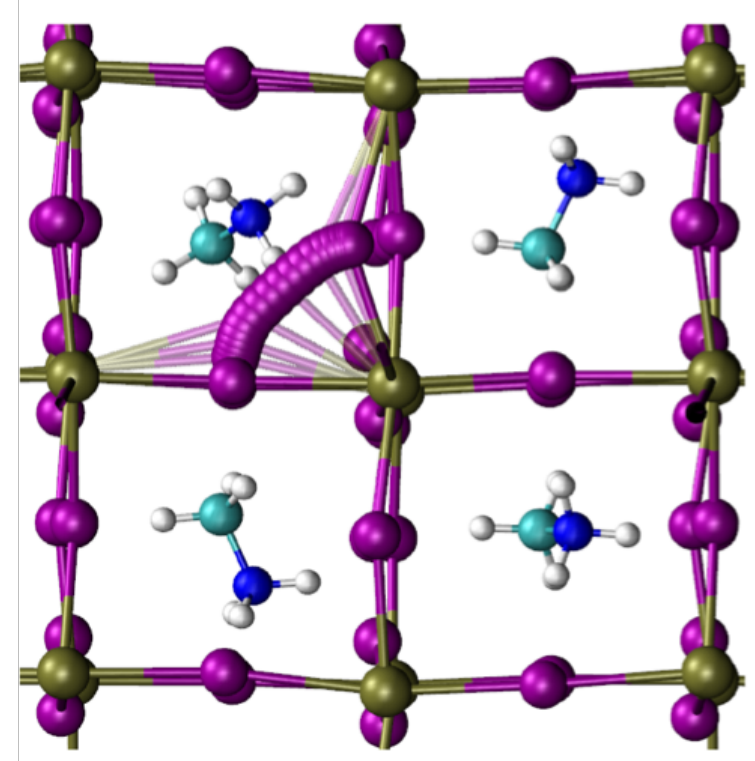

a

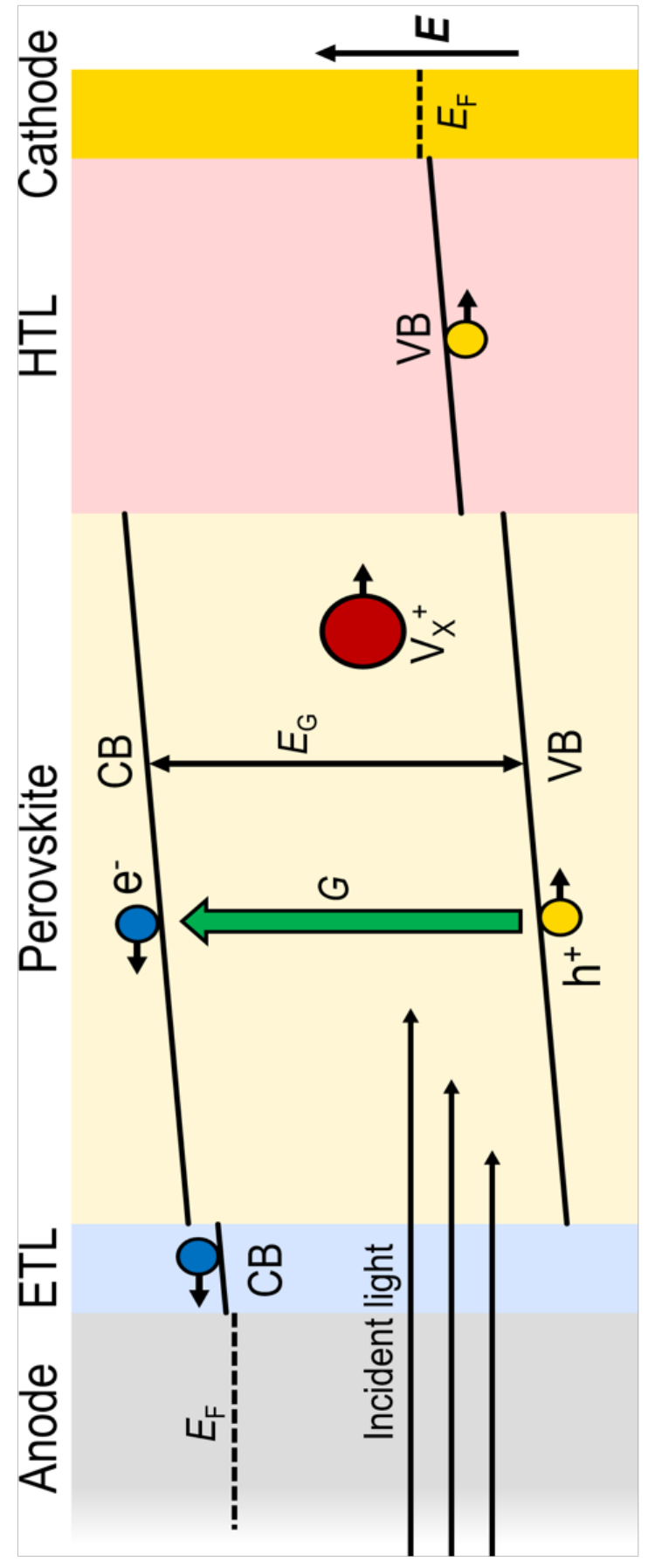

త 

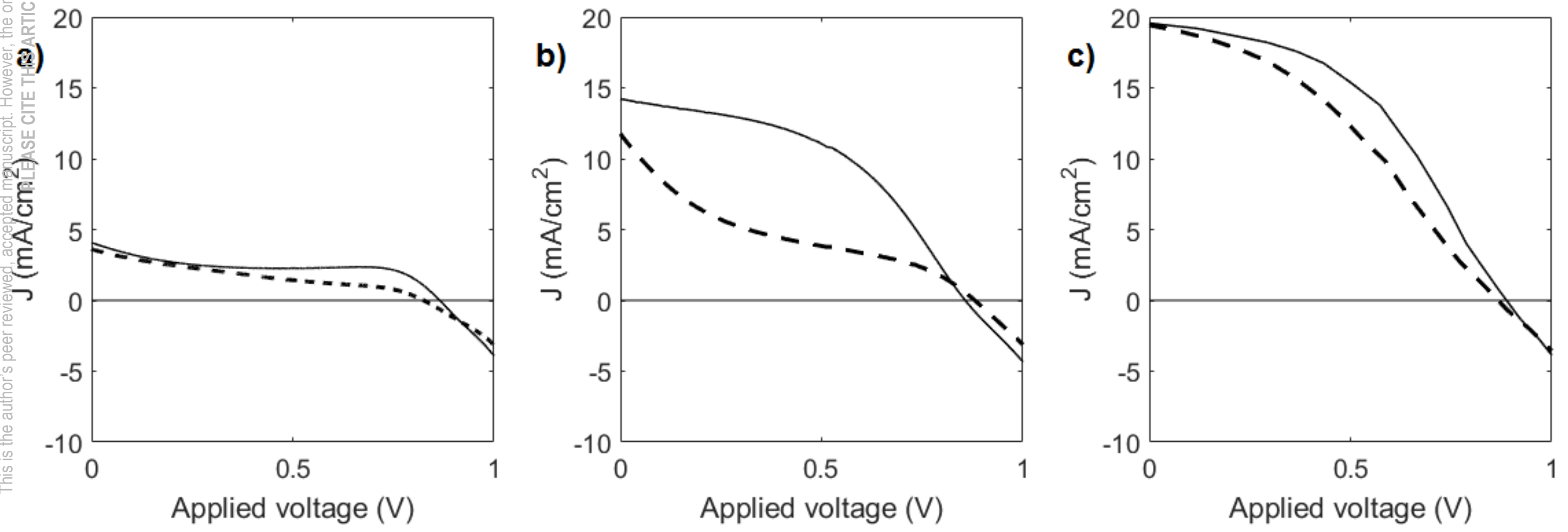
a)

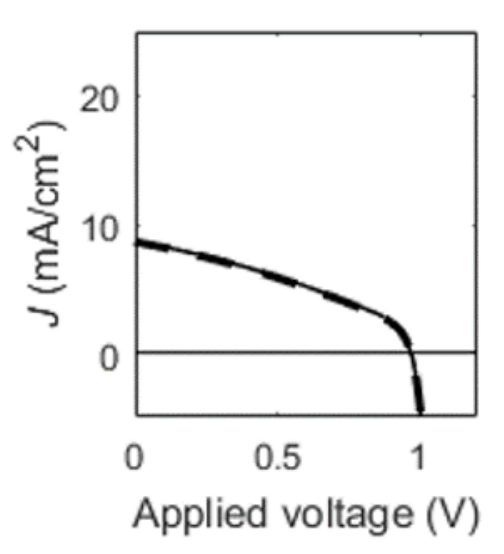

b)

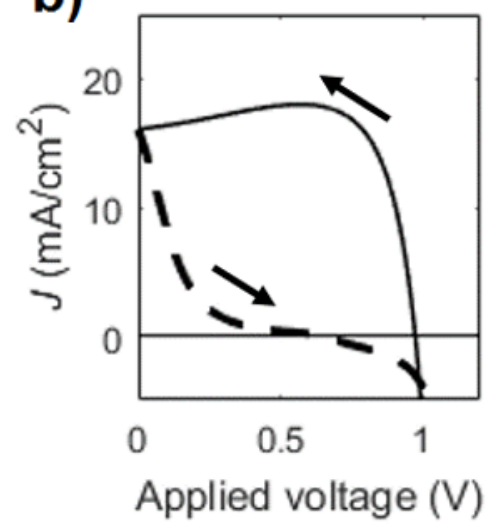

c)

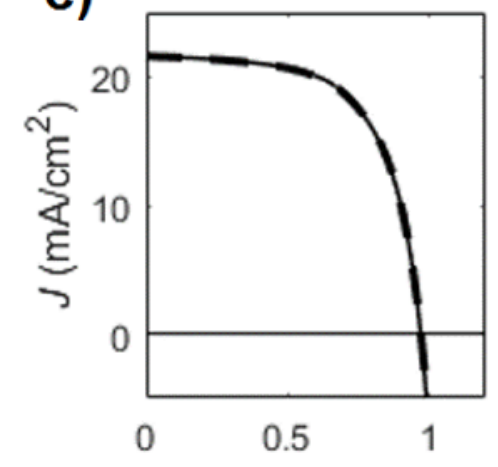

Applied voltage (V)

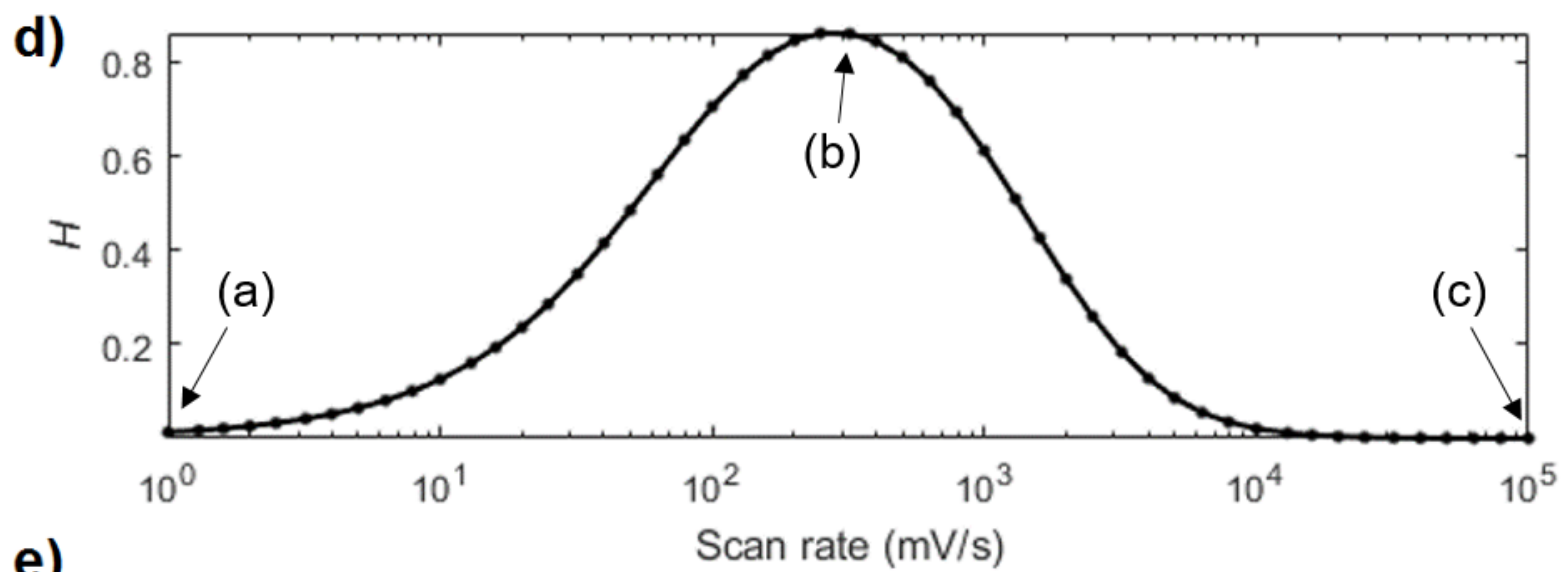

e)

Scan rate $(\mathrm{mV} / \mathrm{s})$

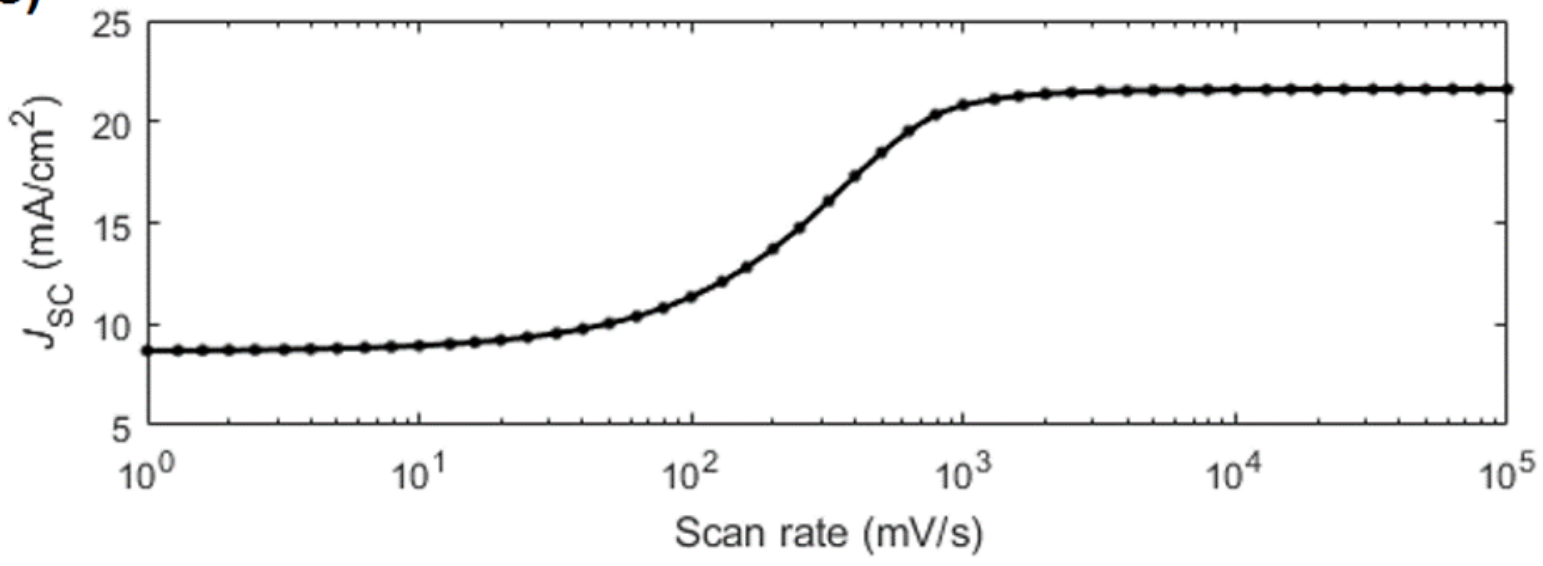



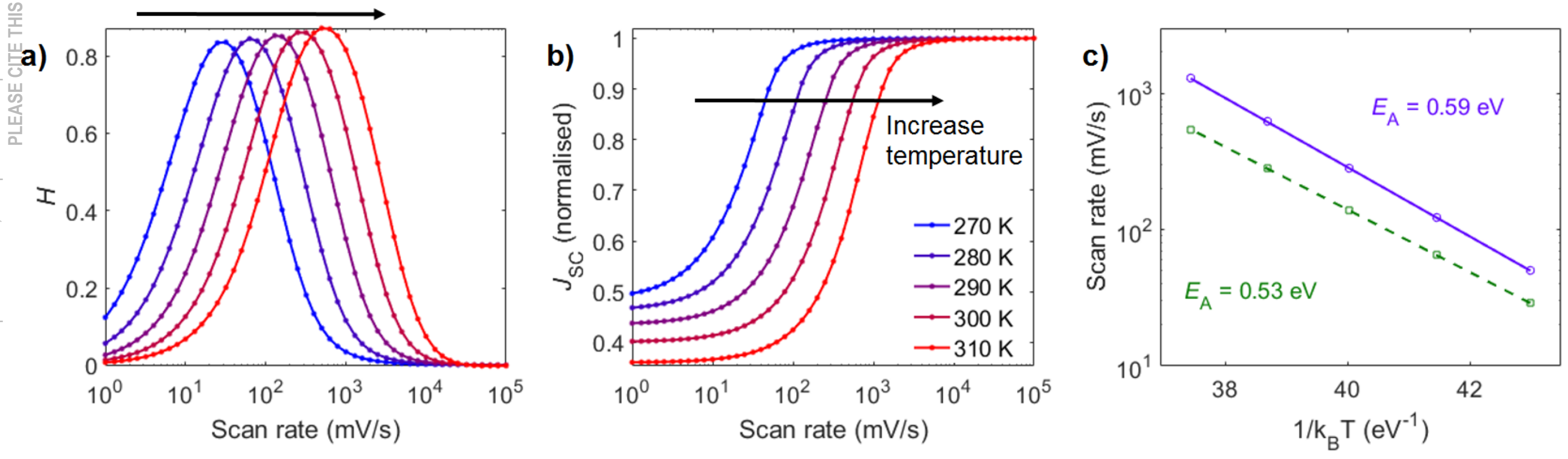
a)

\begin{tabular}{|c|}
\hline $\mathrm{Au}$ \\
\hline spiro \\
\hline $\mathrm{MAPI}$ \\
\hline $\mathrm{TiO}_{2}$ \\
\hline $\mathrm{FTO}$ \\
\hline
\end{tabular}

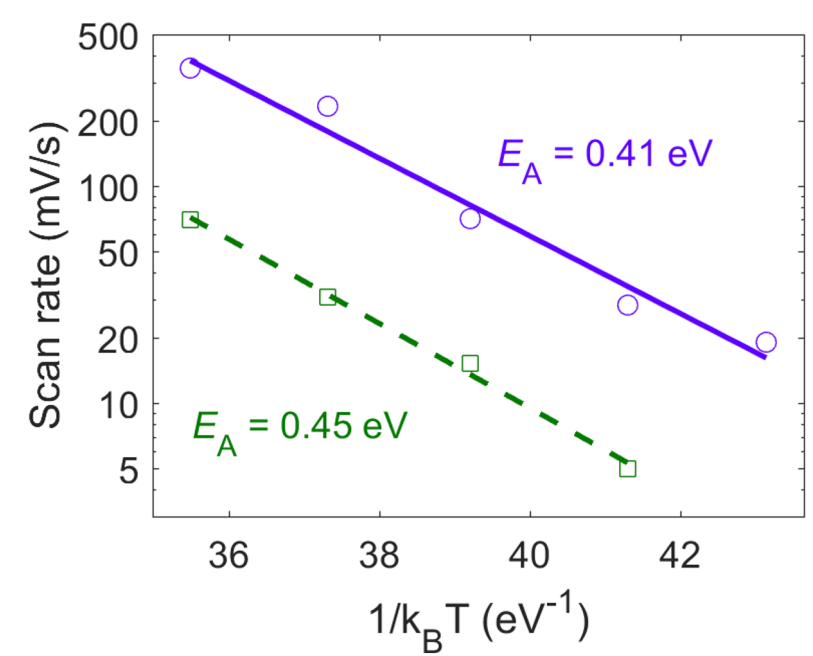

c)

\begin{tabular}{|c|}
\hline $\mathrm{Au}$ \\
\hline spiro \\
\hline $\mathrm{FAPI}$ \\
\hline $\mathrm{SnO}_{2}$ \\
\hline FTO \\
\hline
\end{tabular}

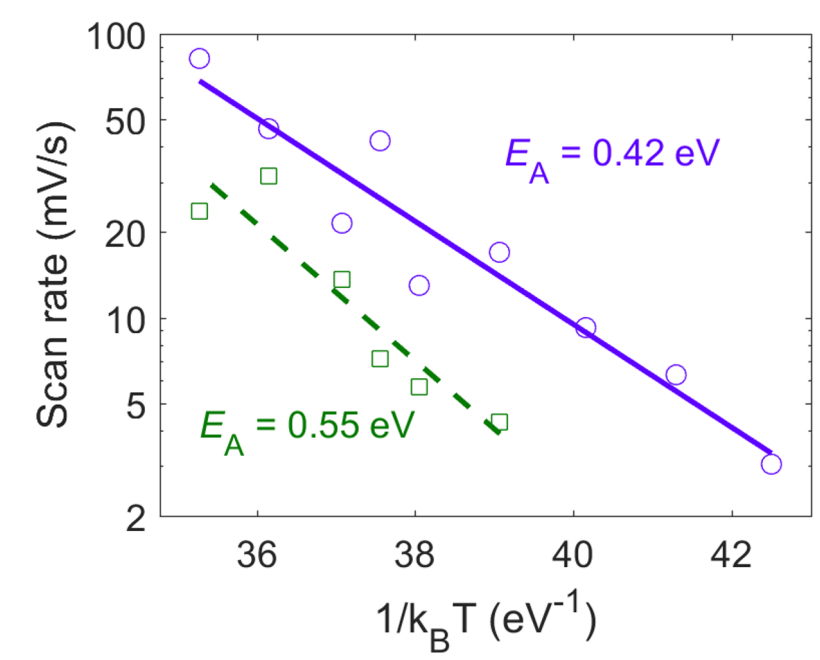

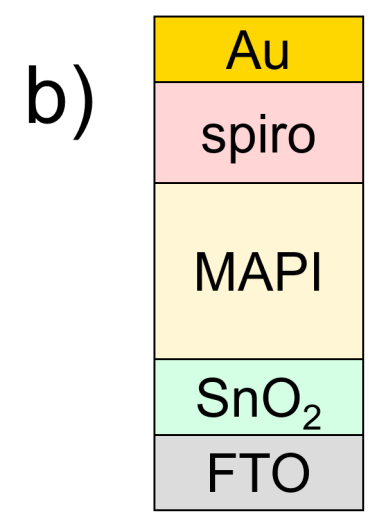
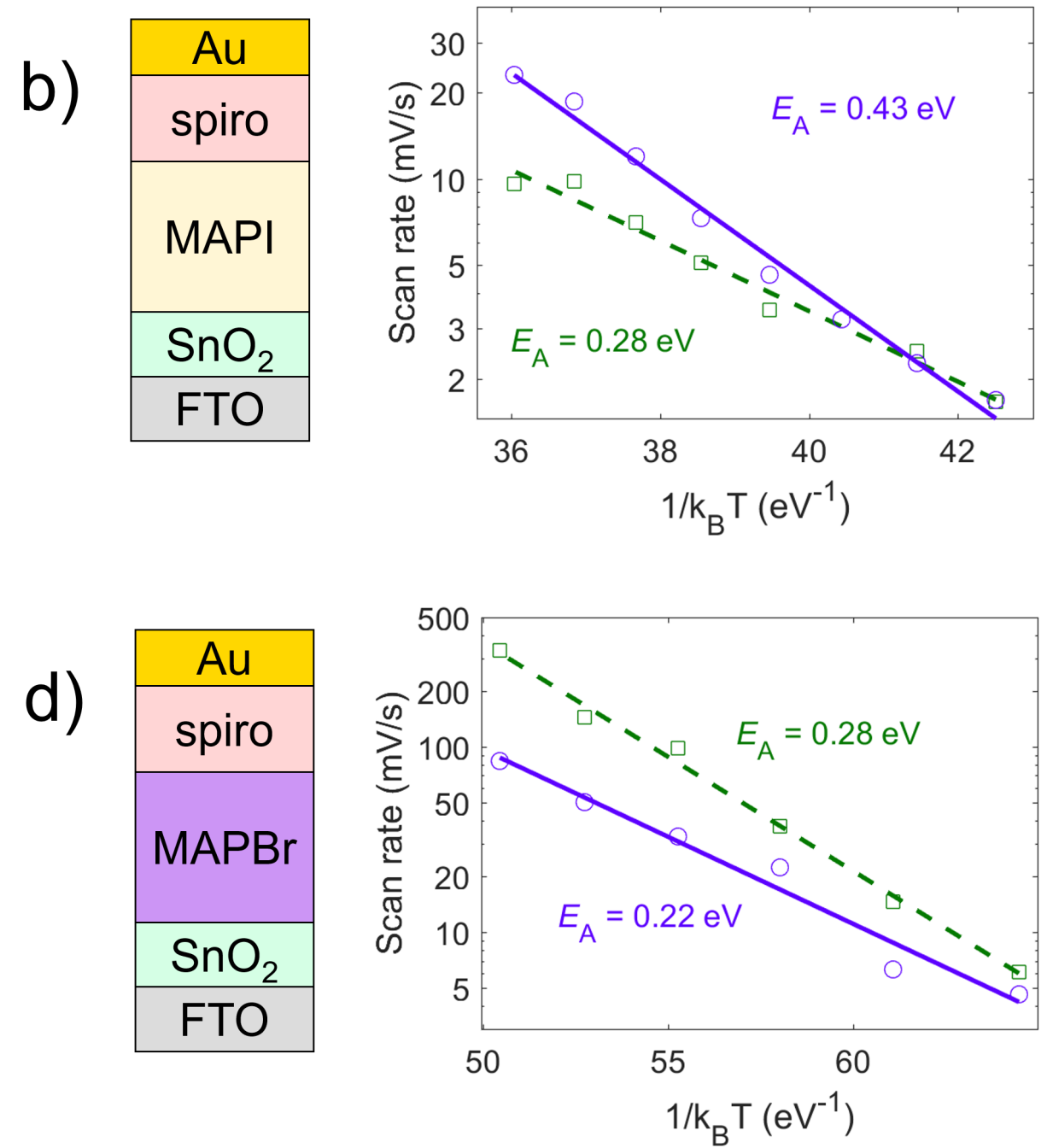

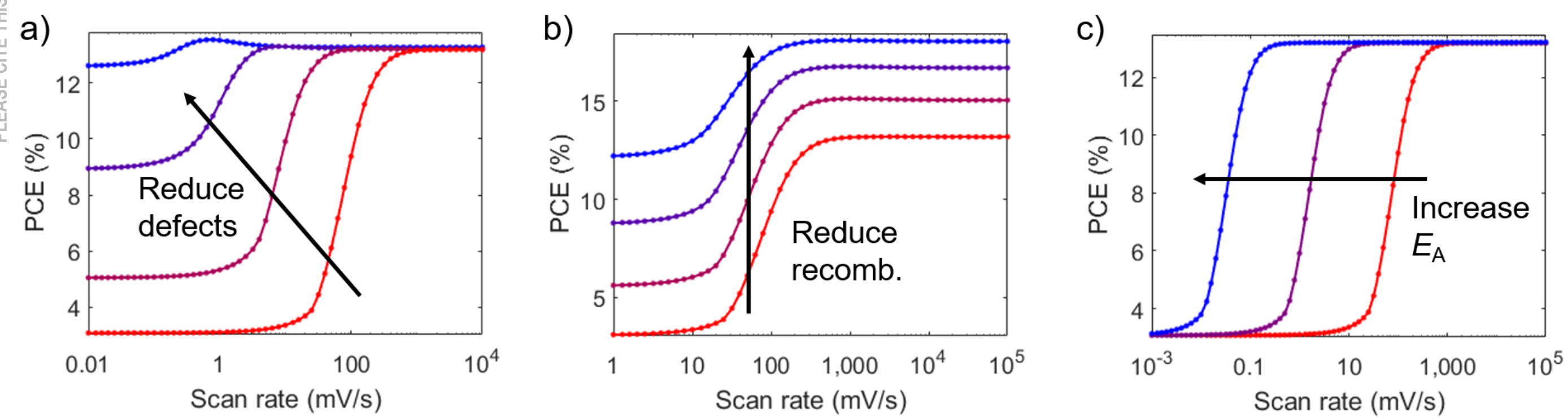